Atherosclerotic lesions were present in the aortae of the $\mathrm{ApoE}^{-/-}$mice. A significant increase in total lesion area occupying the aorta was observed in the aged $\mathrm{ApoE}^{-/-}$mice compared to mice at the earlier time-point $(0.11 \%$ vs. $2.78 \%, \mathrm{P}=$ $0.006, \mathrm{n}=6-7$ mice per group). A significant increase in the amount of PVAT surrounding the aortae of C57/BL6 mice was recorded in response to ageing $(15.65 \pm 2.90 \mathrm{mg}$ versus $26.87 \pm 2.84 \mathrm{mg}, \mathrm{P}=0.02 \mathrm{n}=5-8$ mice per group). In contrast, no differences in the amount of aortic PVAT was observed between young and ageing $\mathrm{ApoE}^{-/-}$mice (14.08 \pm $1.64 \mathrm{mg}$ versus $13.11 \pm 1.78 \mathrm{mg}, \mathrm{P}=\mathrm{NS}, \mathrm{n}=3-5)$. Aortic PVAT exerted anti-contractile effects in the young 8 week controls (PVAT versus no PVAT: $\mathrm{P}=0.04, \mathrm{n}=6$ ). However, the anti-contractile capacity of aortic PVAT was abolished in ageing control mice (PVAT versus no PVAT: $\mathrm{P}=\mathrm{NS}, \mathrm{n}=8$ ). The aortic PVAT from young $\mathrm{ApoE}^{-/-}$mice exhibited the characteristics of an aged phenotype, exerting no anti-contractile effect (PVAT versus no PVAT: $\mathrm{P}=\mathrm{NS}, \mathrm{n}=8$ ); this was sustained in ageing $\mathrm{ApoE}^{-/-}$mice (PVAT versus no PVAT: $\mathrm{P}=\mathrm{NS}$, $\mathrm{n}=8$ ). Modulations in adipokine expression were observed with ageing in both $\mathrm{C} 57 / \mathrm{BL} 6$ and $\mathrm{ApoE}^{-/-}$mice.

Aortic PVAT from ApoE ${ }^{-/-}$mice displayed an aged phenotype even at 12 weeks of age. These findings may have important implications in the pathogenesis of atherosclerosis. Further investigation into the characteristics of aortic PVAT from $\mathrm{ApoE}^{-/-}$mice and the factors it releases in early and established atherosclerosis is ongoing.

This research was funded by the British Heart Foundation.

\section{DIFFERENTIAL EFFECTS OF RESVERATOL ON ACETYLCHOLINE-INDUCED AND FLOW-MEDIATED DILATION OF THE MOUSE FEMORAL ARTERY}

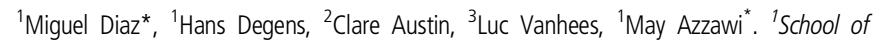
Healthcare Science, Faculty of Science and Engineering, Manchester Metropolitan University, Manchester, UK; ${ }^{2}$ Faculty of Health and Social Care, Edgehill University, Lancashire, UK; ${ }^{3}$ Department of Rehabilitation Sciences, University of Leuven, Belgium University; *Presenting Author
\end{abstract}

\subsection{6/heartjnl-2016-309890.189}

Despite the widespread use of nutritional supplements, insight into their potential beneficial or harmful biological effects is frequently lacking. Resveratrol (RV) is a polyphenol found in berries and grape skin and is often marketed as beneficial for vascular health. Here, we tested this assumption and hypothesised that RV enhances endothelial function and flow-mediated dilation (FMD) in mouse femoral arteries.

C57BL/6 male mice (12 weeks of age) were humanely euthanised in accordance with the $\hat{\mathrm{a}} €$ Animals (Scientific Procedures) Act $1986 \hat{\mathfrak{a}} €^{\mathrm{rм}}$ and Institutional guidelines. Sections of the femoral artery were dissected and mounted between two glass cannulae on a modified pressure myograph chamber. Arteries were pressurised to an intravascular pressure of 60 $\mathrm{mmHg}$. Endothelial-dependent dilator responses to acetylcholine (ACh) and intraluminal flow in pre-constricted vessels (Phe $10^{-5} \mathrm{M}$ ) were assessed in femoral arteries incubated for $1 \mathrm{~h}$ in either $30 \hat{\mathrm{A}} \mu \mathrm{M}$ RV or physiological saline solution (PSS). The contribution of different vasodilator pathways was investigated by treating sections of the femoral artery with LNG-nitro-L-arginine (L-NNA; 100uM) or indomethacin (10 $\hat{A} \mu \mathrm{M})$. The drugs were applied intraluminally for $30 \mathrm{~min}$ using a $1-\mathrm{mL}$ syringe inserted into one end of a $3 \hat{a} €$ way luer connexion on the side of the pressure myograph chamber while incubated in PSS or RV. After 30 min incubation, dilator responses to $\mathrm{ACh}\left(10^{-9}-10^{-3} \mathrm{M}\right)$ and intraluminal flow $(5-10$ $\left.\hat{A} \mu \mathrm{LA} \cdot \mathrm{min}^{-1}\right)$ in the presence or absence of these drugs were determined.

Maximal degrees of dilation were reached upon perfusing the arteries with of $10^{-5}$ to $10^{-3} \mathrm{M}$ ACh. RV treatment significantly enhanced dilation in response to $\mathrm{ACh}(\mathrm{p}<0.05)$. Whereas dilation of the arteries in response to ACh was $($ mean \pm SEM) $68.1 \pm 13.7 \%(n=9)$ of the passive diameter when incubated with PSS, treatment with RV resulted in dilation of $92.2 \pm 13.4 \%(n=6)$. However, RV significantly reduced FMD ( $\mathrm{p}<0.05)$; dilation in response to intraluminal flow of $8 \hat{\mathrm{A}} \mu \mathrm{LA} \cdot \mathrm{min}^{-1}$ was $22.5 \pm 7.1 \%(\mathrm{n}=9)$ and $0.67 \pm$ $1.06 \%(\mathrm{n}=6)$ of the passive when the sections were incubated with PSS and RV, respectively. Incubation with L-NNA reduced dilation to $\mathrm{ACh}$ in the presence of RV. However, the effects of RV on ACh dilation were maintained in the presence of indomethacin. The effects of RV on FMD were not significantly modulated by L-NNA or indomethacin. The responses to intraluminal flow were still compromised and no significant dilation was present. Our data suggest that RV may have differential effects on ACh-dependent and FMD responses in the mouse femoral artery. The use of RV as a nutritional supplement still warrants caution.

\section{INVESTIGATING PLATELET FUNCTIONAL HETEROGENEITY USING DROPLET MICROFLUIDICS}

Maaike Jongen*, Nicola Englyst, Jonathan West. University of Southampton; *Presenting Author

\subsection{6/heartjnl-2016-309890.190}

Platelet activation is an important step in arterial thrombosis, the acute complication of atherosclerosis. However, current diagnostic techniques for platelet function have been shown to be inadequate to predict thrombosis. In spite of this, many patients are prescribed aspirin to prevent (further) occurrence of arterial thrombosis, introducing bleeding risk. Platelets have been shown to be heterogeneous in a number of features such as size, volume and density, and this variety may underpin overall system behaviour. Functional heterogeneity has been suggested in several studies but current methods are not suitable to reliably study single platelet function. Such a method should provide a high throughput means to profile large platelet populations for the identification of, potentially rare, hyperactive platelets. In addition, the technique must prevent paracrine signalling (platelet-mediated activation of neighbouring platelets), necessitating the isolation of single platelets. This study adapts a droplet microfluidics approach to investigate single platelet functionality.

Here, single platelet sensitivity is studied by adding the agonist (convulxin, specific ligand of the GPVI receptor for collagen) during encapsulation in droplets. After an incubation period the platelets are retrieved from the droplets into fixative, followed by flow cytometry analysis of markers for activation. The PAC-1 antibody is used to identify the active conformation of the $\hat{\mathrm{I}} \pm \mathrm{IIb}^{2} 3$ receptor, important for aggregation and adhesion, and anti-CD62P (p-selectin) to identify degranulation of the platelets. Platelets are identified with CD42b, which is a platelet specific receptor. 
Platelets are individually encapsulated in monodisperse (CV of $1-4 \%)$ water-in-oil droplets with a mean volume of $14 \mathrm{pL}$ and a diameter of $30 \quad \hat{\mathrm{I}} 1 / 4 \mathrm{~m}$. Droplets are produced with a throughput of $4 \mathrm{kHz}$, with droplets containing a single platelet produced at a rate of $0.25 \mathrm{kHz}$ (following a Poisson distribution). With this method an intrinsic variation in the platelet response to convulxin is observed, that is unrelated to the size of the platelet.

The research entailed the development of a method capable of measuring the intrinsic variation in platelet function. The method will be used to compare the sensitivity profiles of people with known risk factors for arterial thrombosis with the profiles of people with low risk. This presents the possibility to identify a novel prognostic biomarker. Furthermore, a better understanding of the functional heterogeneity of platelets could be used to identify new targets to aid the rational design of new therapeutics. Ideally, this would target only hyperactive platelets, while preserving normal haemostasis.

\section{ATHEROPRONE FLOW ALTERS ATP-INDUCED CALCIUM SIGNALLING IN THE ENDOTHELIUM}

${ }^{1}$ Jack Green*, ${ }^{2}$ Paul Evans, ${ }^{2}$ Heather Wilson. 'University of Sheffield; ${ }^{2}$ Department of Infection, Immunity and Cardiovascular Disease (IICD), University of Sheffield; *Presenting Author

\subsection{6/heartjnl-2016-309890.191}

Introduction The development of the atherosclerotic plaque occurs at distinct points in the vasculature, with areas such as bends and branch points susceptible to plaque formation. The endothelium at these sites is influenced by a disturbed blood flow with low wall shear stress. Numerous studies have shown that stimulation of endothelial cells with this flow pattern induces several inflammatory signalling pathways, but the mechanisms in which this flow pattern is sensed remain unclear.

ATP is released extracellularly from endothelial cells in response to shear stress. The endothelium expresses the ATPgated cation channels P2X4 and P2X7, which have been previously shown to respond to shear stress induced ATP release. Their expression is also increased in the endothelium following inflammatory stimulation. Therefore, we hypothesise that P2X receptors are involved in sensing atheroprone flow and inducing inflammatory signalling, thereby promoting development of atherosclerosis.

Methods Human Umbilical Vein Endothelial Cells (HUVECs) were cultured under flow for $72 \mathrm{~h}$ using an ibidi flow pump system. Atheroprotective flow was applied using $+13 \mathrm{dyn} / \mathrm{cm}^{2}$ and $\pm 4 \mathrm{dyn} / \mathrm{cm}^{2}(0.5 \mathrm{hz})$ was used for atheroprone flow. After flow conditioning, HUVECs were then loaded with a fluorescent calcium dye (CAL-520) and their response to

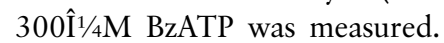

Results Endothelial cells preconditioned with atheroprone flow showed an enhanced calcium response after BzATP stimulation. This enhancement was dependent on extracellular calcium as no increase was detected when performed in calcium free extracellular buffer. The expression level of P2X4 and P2X7 receptors was significantly enhanced in HUVECs preconditioned with atheroprone flow compared to atheroprotective. Moreover the expression of the cell surface ATPase CD39, which breaks down extracellular ATP, was increased under atheroprotective flow. Chemical inhibition of CD39 with ARL-67156 also significantly enhanced the calcium response to BzATP in cells under atheroprotective, but not atheroprone flow.

Conclusions We have identified an enhanced ATP induced calcium response and expression of the ATP-gated P2X4/7 receptors in HUVEC under atheroprone flow conditions. We observed enhanced expression of the cell surface ATPase CD39 in endothelial cells under atheroprotective flow suggesting that extracellular ATP is more rapidly hydrolysed to adenosine under such conditions. We therefore propose that shear stress induced ATP plays a role in specifically activating cells under atheroprone flow and induces inflammatory signalling pathways, underlying atherogenesis susceptibility in vulnerable regions of the vasculature.

\section{HYPOXIA ENHANCES THE REPARATIVE EFFECT OF TISSUE PROTECTIVE ERYTHROPOIETIN AND ITS NON- ERYTHROPOIETIC PEPTIDE ANALOGUE IN AN ENDOTHELIAL CELL INJURY MODEL}

${ }^{1}$ Lamia Heikal ${ }^{*},{ }^{1}$ Pietro Ghezzi, ${ }^{1}$ Manuela Mengozzi, ${ }^{2}$ Martin Feelisch, ${ }^{1}$ Gordon Ferns ${ }^{1}$ University of Sussex; ${ }^{2}$ Faculty of Medicine, University of Southampton; *Presenting Author

\subsection{6/heartjnl-2016-309890.192}

Background Endothelial injury is a critical feature in the early stages of vascular disease. Inflammation and hypoxia are often associated with endothelial injury, stimulating the expression of several cytokines that include erythropoietin (EPO). Endothelial cell-derived EPO appears to be important for protecting the endothelium against ischaemic injury. A non-erythropoietic analogue of EPO; pyroglutamate helix B surface peptide (pHBSP) retains these protective properties of EPO without possessing its erythropoietic effects. The aim of our study was to assess the effects of these molecules in a model of endothelial injury under normoxic and hypoxic conditions.

Method The reparative effects of EPO and pHBSP were assessed under hypoxia $\left(1 \% \mathrm{O}_{2}\right)$ and normoxia $\left(21 \% \mathrm{O}_{2}\right)$ in an in vitro model of endothelial injury (scratch assay). A monolayer of bovine aortic endothelial cells (BAECs), grown to confluence in a multi-well plate, was scratched and the closure of the injured endothelial monolayer was assessed over $24 \mathrm{~h}$. The effects of EPO and pHBSP on BAEC proliferation, chemotaxis and apoptosis were assessed under similar hypoxic conditions in separate experiments. The potential molecular mechanisms of these effects were also explored.

Results Both EPO and pHBSP enhanced scratch closure under hypoxic conditions by $13 \pm 2.6 \%$, and $10 \pm 1.69 \%$ respectively $(\mathrm{p}<0.01)$ compared to normoxic conditions $(3.2 \pm$ $0.9 \%$ and $2.9 \pm 0.3 \%$ for EPO and pHBSP respectively $\mathrm{p}>$ $0.05)$. These effects appeared to be by promoting cell proliferation and migration of BAECs $(\mathrm{p}<0.05)$. EPO also protected BAECs from staurosporine-induced apoptosis under hypoxic conditions. The priming effect of hypoxia was associated with

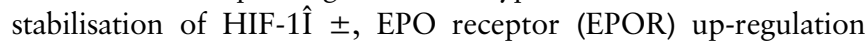
and decreased phosphorylation of endothelial nitric oxide synthase (eNOS) at the Ser-1177 residue. The effect of hypoxia on the latter was rescued by EPO. Hypoxia was associated with a reduction in nitric oxide (NO) production as assessed by its oxidation products nitrite and nitrate, and this was consistent with the oxygen requirement for the endogenous production of NO by NO synthase (NOS). Whilst EPO did not affect NO formation in normoxia, it markedly increased NO production under hypoxic conditions, in a NOS-dependent 